\title{
Direct Positioning in Synchronous and Asynchronous Visible Light Systems
}

\author{
Musa Furkan Keskin, Sinan Gezici, and Orhan Arikan \\ Department of Electrical and Electronics Engineering, Bilkent University, 06800, Ankara, Turkey \\ Emails: \{keskin,gezici,oarikan\}@ee.bilkent.edu.tr
}

\begin{abstract}
In this paper, direct positioning approaches are investigated for both synchronous and asynchronous visible light systems. First, the maximum likelihood estimator (MLE) and the Cramér-Rao lower bound (CRLB) are derived for the synchronous scenario by considering the information from both channel attenuation factors and time delay parameters. Then, asynchronous visible light positioning (VLP) systems are investigated and the corresponding CRLBs and MLEs are obtained based on received signal strength (RSS) information. It is shown that the direct and two-step position estimators are equivalent in the asynchronous scenario under mild conditions. Numerical examples are provided to illustrate the theoretical results.

Index Terms- Estimation, Cramér-Rao lower bound, visible light, Lambertian pattern, direct positioning.
\end{abstract}

\section{INTRODUCTION}

Light emitting diode (LED) based visible light systems are well suited for communication and localization in indoor environments due to their attractive property of simultaneous illumination, high speed communications, and positioning [1], [2]. Unlike radio frequency (RF) based indoor localization services, visible light positioning (VLP) systems can utilize an enormous unregulated visible light spectrum without suffering from interference by RF communications and other LED radiation sources [1]. In addition, high accuracy localization results have been demonstrated for VLP systems in the literature; e.g., [3], [4]. The aim of this paper is to establish a theoretical framework for direct positioning in both synchronous and asynchronous VLP systems with arbitrary transmitter/receiver orientations.

In the literature, the problem of wireless localization has been treated by employing two classes of approaches, which can be regarded as two-step positioning and direct positioning. Widely applied in RF localization and VLP systems, twostep positioning algorithms extract position related parameters, such as received signal strength (RSS), time-of-arrival (TOA), time-difference-of-arrival (TDOA), and angle-of-arrival (AOA) in the first step, and perform position estimation based on those parameters in the second step [5]. There exist a multitude of applications of indoor VLP systems employing two-step positioning, such as those using RSS [4], [6], AOA [7], hybrid RSS/AOA [8]-[10], TOA [11], [12] and TDOA [13]. However, the two-step method can be regarded as a suboptimal solution to the localization problem in general since it does not exploit all the collected data related to the unknown location. On the other hand, direct positioning algorithms are based on utilizing the entire received signal in a one-step process in order to determine the unknown position, as opposed to twostep positioning [14], [15].
Although the direct positioning approach has been employed in numerous applications in RF based wireless localization [14], [16]-[19] and radar systems [20], [21], only a limited amount of research has been carried out on the utilization of direct positioning for VLP systems. In [22], an RSS based VLP system is proposed where time-averaged RSS values at each photodetector (PD) are employed as the final observation for position estimation. An asynchronous VLP system is designed in [23], where a Bayesian signal model is constructed to estimate the unknown position based on the entire received signal from multiple LEDs. In addition, there exist several studies that focus on theoretical bounds for ranging and positioning in VLP systems. The CRLB for distance estimation is derived for synchronous [11], [12] and asynchronous [24] VLP systems. In [9], the CRLB is derived for RSS based three-dimensional localization for an indoor VLP scenario with arbitrary configurations of LED transmitters and visible light communication (VLC) receivers. In [22], an analytical CRLB expression is derived for twodimensional RSS-based positioning with a known receiver height. As a novel contribution to visible light based indoor localization, the CRLB derivations presented in this paper take into account both the TOA and RSS information and consider raw measurements (i.e., the entire observed signal at the VLC receiver, not the extracted parameters) as an input to the positioning algorithm.

In this paper, theoretical accuracy limits and corresponding maximum likelihood estimators (MLEs) are derived for three-dimensional localization of a VLC receiver in both synchronous and asynchronous VLP systems. The proposed theoretical bounds and estimators are generic and applicable to any indoor VLP localization scenario. In addition, it is obtained that direct and two-step position estimators are equivalent for asynchronous VLP systems. The derived CRLB expressions differ from those for VLP systems [9], [22] in the sense that (i) they incorporate time delay information by considering also the synchronous scenario and (ii) derivations are established based directly on the entire received signal, not on the measured/extracted parameters. In addition, the provided bounds have significant differences from those obtained for RF localization systems [14], [15], [19], [20]. The reason is twofold: (i) both TOA and RSS information are used in the considered synchronous VLP system whereas only TOA information is utilized in synchronous RF systems (RSS information is not reliable due to multipath effects) and (ii) Lambertian equation for the channel attenuation factor is available in terms of system parameters for VLP systems whereas no such formula exists for RF systems. 
The rest of the paper is organized as follows: Section II presents the VLP system model. CRLBs and MLEs are derived for synchronous and asynchronous systems in Section III and Section IV, respectively. Numerical results are presented in Section V and concluding remarks are provided in Section VI.

\section{System Model}

\section{A. Received Signal Model}

Consider a VLP system in which a number of LED transmitters are employed to estimate the position of a VLC receiver. A line-of-sight (LOS) scenario is assumed between each LED transmitter and the VLC receiver, which is commonly the case for visible light systems [2], [11]. Then, the received signal at the VLC receiver due to the signal emitted by the $i$ th LED transmitter is formulated as [11]

$$
r_{i}(t)=\alpha_{i} R_{p} s_{i}\left(t-\tau_{i}\right)+\eta_{i}(t)
$$

for $i \in\left\{1, \ldots, N_{\mathrm{L}}\right\}$ and $t \in\left[T_{1, i}, T_{2, i}\right]$, where $N_{\mathrm{L}}$ denotes the number of LED transmitters, $T_{1, i}$ and $T_{2, i}$ determine the observation interval for the signal coming from the $i$ th LED transmitter, $\alpha_{i}$ is the attenuation factor of the optical channel between the $i$ th LED transmitter and the VLC receiver $\left(\alpha_{i}>0\right), R_{p}$ is the responsivity of the photo detector, $s_{i}(t)$ is the transmitted signal from the $i$ th LED transmitter, which is nonzero over an interval of $\left[0, T_{s, i}\right], \tau_{i}$ is the time-ofarrival (TOA) of the signal emitted by the $i$ th LED transmitter, and $\eta_{i}(t)$ is zero-mean additive white Gaussian noise with spectral density level $\sigma^{2}$. It is assumed that a certain type of multiple access protocol, such as frequency-division or timedivision multiple access, is employed in order to facilitate separate processing of signals from each LED transmitter at the VLC receiver [25]. The noise processes corresponding to the received signals from different LED transmitters are supposed to be independent. It is also assumed that $R_{p}$ and $s_{i}(t), i \in\left\{1, \ldots, N_{\mathrm{L}}\right\}$, are known by the VLC receiver.

The TOA parameter in (1) is modeled as

$$
\tau_{i}=\frac{\left\|\boldsymbol{l}_{\mathrm{r}}-\boldsymbol{l}_{\mathrm{t}}^{i}\right\|}{c}+\Delta_{i}
$$

where $c$ is the speed of light, $\Delta_{i}$ denotes the time offset between the clocks of the $i$ th LED transmitter and the VLC receiver, $l_{\mathrm{r}}=\left[\begin{array}{llll}l_{\mathrm{r}, 1} & l_{\mathrm{r}, 2} & l_{\mathrm{r}, 3}\end{array}\right]^{T}$ and $\boldsymbol{l}_{\mathrm{t}}^{i}=\left[\begin{array}{lll}l_{\mathrm{t}, 1}^{i} & l_{\mathrm{t}, 2}^{i} & l_{\mathrm{t}, 3}^{i}\end{array}\right]^{T}$ are three-dimensional column vectors that denote the locations of the VLC receiver and the $i$ th LED transmitter, respectively, and $\left\|\boldsymbol{l}_{\mathrm{r}}-\boldsymbol{l}_{\mathrm{t}}^{i}\right\|$ denotes the distance between the $i$ th LED transmitter and the VLC receiver. For a synchronous scenario, $\Delta_{i}=0$ for $i=1, \ldots, N_{\mathrm{L}}$, whereas for an asynchronous scenario, $\Delta_{i}$ 's are modeled as deterministic unknown parameters. It is assumed that the signal component in (1) is contained completely in the observation interval $\left[T_{1, i}, T_{2, i}\right]$; that is, $\tau_{i} \in\left[T_{1, i}, T_{2, i}-T_{s, i}\right]$. In (1), the channel attenuation factor $\alpha_{i}$ is modeled as

$$
\alpha_{i}=-\frac{\left(m_{i}+1\right) S}{2 \pi} \frac{\left[\left(\boldsymbol{l}_{\mathrm{r}}-\boldsymbol{l}_{\mathrm{t}}^{i}\right)^{T} \boldsymbol{n}_{\mathrm{t}}^{i}\right]^{m_{i}}\left(\boldsymbol{l}_{\mathrm{r}}-\boldsymbol{l}_{\mathrm{t}}^{i}\right)^{T} \boldsymbol{n}_{\mathrm{r}}}{\left\|\boldsymbol{l}_{\mathrm{r}}-\boldsymbol{l}_{\mathrm{t}}^{i}\right\|^{m_{i}+3}}
$$

where $m_{i}$ is the Lambertian order for the $i$ th LED transmitter, $S$ is the area of the photo detector at the VLC receiver, and $\boldsymbol{n}_{\mathrm{r}}=\left[\begin{array}{lll}n_{\mathrm{r}, 1} & n_{\mathrm{r}, 2} & n_{\mathrm{r}, 3}\end{array}\right]^{T}$ and $\boldsymbol{n}_{\mathrm{t}}^{i}=\left[\begin{array}{lll}n_{\mathrm{t}, 1}^{i} & n_{\mathrm{t}, 2}^{i} & n_{\mathrm{t}, 3}^{i}\end{array}\right]^{T}$ denote the orientation vectors ('normals') of the VLC receiver and the $i$ th LED transmitter, respectively [9], [11]. ${ }^{1}$ It is assumed that the VLC receiver knows $S, \boldsymbol{n}_{\mathrm{r}}, m_{i}, \boldsymbol{l}_{\mathrm{t}}^{i}$, and $\boldsymbol{n}_{\mathrm{t}}^{i}$ for $i=1, \ldots, N_{\mathrm{L}}$. For example, the orientation of the VLC receiver, $\boldsymbol{n}_{\mathrm{r}}$, can be determined by a gyroscope and the parameters of the LED transmitters $\left(m_{i}, \boldsymbol{l}_{\mathrm{t}}^{i}\right.$ and $\left.\boldsymbol{n}_{\mathrm{t}}^{i}\right)$ can be sent via VLC to the receiver.

\section{B. Log-Likelihood Function and CRLB}

Considering the received signal model in (1), the loglikelihood function for the received signal vector $\boldsymbol{r}(t) \triangleq$ $\left[r_{1}(t) \ldots r_{N_{\mathrm{L}}}(t)\right]^{T}$ is obtained as follows [26], [27]:

$$
\Lambda(\boldsymbol{\varphi})=k-\frac{1}{2 \sigma^{2}} \sum_{i=1}^{N_{\mathrm{L}}} \int_{T_{1, i}}^{T_{2, i}}\left(r_{i}(t)-\alpha_{i} R_{p} s_{i}\left(t-\tau_{i}\right)\right)^{2} d t
$$

where $\varphi$ represents the set of unknown parameters and $k$ is a normalizing constant that does not depend on the unknown parameters. While the set of unknown parameters consists only of the coordinates of the VLC receiver in the synchronous case, it also contains the delay parameters in the asynchronous case, as investigated in Sections III and IV.

The CRLB on the covariance matrix of any unbiased estimator $\hat{\varphi}$ of $\varphi$ can be expressed as [28]

$$
\mathbb{E}\left\{(\hat{\varphi}-\varphi)(\hat{\varphi}-\varphi)^{T}\right\} \succeq \mathbf{J}(\varphi)^{-1}
$$

where $\boldsymbol{A} \succeq \boldsymbol{B}$ means that $\boldsymbol{A}-\boldsymbol{B}$ is positive semidefinite and $\mathbf{J}(\varphi)$ is the Fisher information matrix (FIM) for $\varphi$, which can be calculated as follows:

$$
\mathbf{J}(\varphi)=\mathbb{E}\left\{\left(\nabla_{\varphi} \Lambda(\varphi)\right)\left(\nabla_{\varphi} \Lambda(\varphi)\right)^{T}\right\}
$$

with $\nabla_{\varphi}$ representing the gradient operator with respect to $\varphi$ and $\Lambda(\varphi)$ being the log-likelihood function as defined in (4).

\section{Positioning In Synchronous Systems}

In the synchronous scenario, the VLC receiver is synchronized with the LED transmitters; that is, $\Delta_{i}=0$ in (2) for $i=1, \ldots, N_{\mathrm{L}}$. In this section, the CRLB is derived for synchronous VLP systems, and the direct position estimation is proposed by considering both time delay and channel attenuation information.

\section{A. $C R L B$}

In the synchronous case, $\alpha_{i}$ and $\tau_{i}$ are functions of $\boldsymbol{l}_{\mathrm{r}}$ only (since $\Delta_{i}=0$ in (2)); hence, the set of unknown parameters in (4) is defined as

$$
\boldsymbol{\varphi}=\left[\begin{array}{lll}
l_{\mathrm{r}, 1} & l_{\mathrm{r}, 2} & l_{\mathrm{r}, 3}
\end{array}\right]^{T}=\boldsymbol{l}_{\mathrm{r}} .
$$

Then, the CRLB for estimating $\boldsymbol{l}_{\mathrm{r}}$ based on $r_{1}(t), \ldots, r_{N_{\mathrm{L}}}(t)$ in (1) is specified by the following proposition.

Proposition 1: For synchronous VLP systems, the CRLB on the MSE of any unbiased estimator $\hat{l}_{\mathrm{r}}$ for the location of the $V L C$ receiver is given by

$$
\mathbb{E}\left\{\left\|\hat{\boldsymbol{l}}_{\mathrm{r}}-\boldsymbol{l}_{\mathrm{r}}\right\|^{2}\right\} \geq \operatorname{trace}\left\{\mathbf{J}_{\mathrm{syn}}^{-1}\right\}
$$

${ }^{1}$ For example, if the VLC receiver is pointing up directly, then $\boldsymbol{n}_{\mathrm{r}}=$ $\left[\begin{array}{lll}0 & 0 & 1\end{array}\right]^{T}$. 
where

$$
\begin{aligned}
{\left[\mathbf{J}_{\mathrm{syn}}\right]_{k_{1}, k_{2}}=} & \frac{R_{p}^{2}}{\sigma^{2}} \sum_{i=1}^{N_{\mathrm{L}}}\left(E_{2}^{i} \frac{\partial \alpha_{i}}{\partial l_{\mathrm{r}, k_{1}}} \frac{\partial \alpha_{i}}{\partial l_{\mathrm{r}, k_{2}}}+E_{1}^{i} \alpha_{i}^{2} \frac{\partial \tau_{i}}{\partial l_{\mathrm{r}, k_{1}}} \frac{\partial \tau_{i}}{\partial l_{\mathrm{r}, k_{2}}}\right. \\
& \left.-E_{3}^{i} \alpha_{i}\left(\frac{\partial \alpha_{i}}{\partial l_{\mathrm{r}, k_{1}}} \frac{\partial \tau_{i}}{\partial l_{\mathrm{r}, k_{2}}}+\frac{\partial \tau_{i}}{\partial l_{\mathrm{r}, k_{1}}} \frac{\partial \alpha_{i}}{\partial l_{\mathrm{r}, k_{2}}}\right)\right)
\end{aligned}
$$

for $k_{1}, k_{2} \in\{1,2,3\}$ with

$$
\begin{aligned}
E_{1}^{i} & \triangleq \int_{0}^{T_{s, i}}\left(s_{i}^{\prime}(t)\right)^{2} d t \\
E_{2}^{i} & \triangleq \int_{0}^{T_{s, i}}\left(s_{i}(t)\right)^{2} d t \\
E_{3}^{i} & \triangleq \int_{0}^{T_{s, i}} s_{i}(t) s_{i}^{\prime}(t) d t \\
\frac{\partial \tau_{i}}{\partial l_{\mathrm{r}, k}} & =\frac{l_{\mathrm{r}, k}-l_{\mathrm{t}, k}^{i}}{c\left\|\boldsymbol{l}_{\mathrm{r}}-\boldsymbol{l}_{\mathrm{t}}^{i}\right\|} \\
\frac{\partial \alpha_{i}}{\partial l_{\mathrm{r}, k}} & =-\frac{\left(m_{i}+1\right) S}{2 \pi}\left(\frac{\left(\left(\boldsymbol{l}_{\mathrm{r}}-\boldsymbol{l}_{\mathrm{t}}^{i}\right)^{T} \boldsymbol{n}_{\mathrm{t}}^{i}\right)^{m_{i}-1}}{\left\|\boldsymbol{l}_{\mathrm{r}}-\boldsymbol{l}_{\mathrm{t}}^{i}\right\|^{m_{i}+3}}\right. \\
& \times\left(m_{i} n_{\mathrm{t}, k}^{i}\left(\boldsymbol{l}_{\mathrm{r}}-\boldsymbol{l}_{\mathrm{t}}^{i}\right)^{T} \boldsymbol{n}_{\mathrm{r}}+n_{\mathrm{r}, k}\left(\boldsymbol{l}_{\mathrm{r}}-\boldsymbol{l}_{\mathrm{t}}^{i}\right)^{T} \boldsymbol{n}_{\mathrm{t}}^{i}\right) \\
& \left.-\frac{\left(m_{i}+3\right)\left(l_{\mathrm{r}, k}-l_{\mathrm{t}, k}^{i}\right)}{\left\|\boldsymbol{l}_{\mathrm{r}}-\boldsymbol{l}_{\mathrm{t}}^{i}\right\|^{m_{i}+5}}\left(\left(\boldsymbol{l}_{\mathrm{r}}-\boldsymbol{l}_{\mathrm{t}}^{i}\right)^{T} \boldsymbol{n}_{\mathrm{t}}^{i}\right)^{m_{i}}\left(\boldsymbol{l}_{\mathrm{r}}-\boldsymbol{l}_{\mathrm{t}}^{i}\right)^{T} \boldsymbol{n}_{\mathrm{r}}\right) .
\end{aligned}
$$

(The proofs are not presented due to the space limitation.)

The CRLB expression specified by (8)-(14) illustrates the effects of the transmitted signals via the $E_{1}^{i}, E_{2}^{i}$, and $E_{3}^{i}$ terms and the impact of the geometry (configuration) via the $\partial \tau_{i} / \partial l_{\mathrm{r}, k}$ and $\partial \alpha_{i} / \partial l_{\mathrm{r}, k}$ terms. Hence, the theoretical limit on the localization accuracy can be evaluated for any given system based on the provided expression. It should be noted that the CRLB expression in Proposition 1 has not been available in the literature, and provides a theoretical limit for synchronous VLP systems by utilizing information from both channel attenuation factors (RSS) and time delay parameters (TOA). Compared to the CRLB in Proposition 1, those in [11], [12], [24] are for distance estimation only, and that in [9] focuses on RSS based localization.

The CRLB expression in Proposition 1 is generic since the LED transmitters and the VLC receiver can have any locations and orientations and the transmitted signals can be in generic forms. Special cases can easily be obtained from (8)-(14). For example, if the transmitted signals satisfy $s_{i}\left(T_{s, i}\right)=s_{i}(0)$ for $i=1, \ldots, N_{\mathrm{L}}$, then $E_{3}^{i}$ in (12) becomes zero and $\left[\mathbf{J}_{\mathrm{syn}}\right]_{k_{1}, k_{2}}$ in (9) reduces to

$\left[\mathbf{J}_{\mathrm{syn}}\right]_{k_{1}, k_{2}}=\frac{R_{p}^{2}}{\sigma^{2}} \sum_{i=1}^{N_{\mathrm{L}}}\left(E_{2}^{i} \frac{\partial \alpha_{i}}{\partial l_{\mathrm{r}, k_{1}}} \frac{\partial \alpha_{i}}{\partial l_{\mathrm{r}, k_{2}}}+E_{1}^{i} \alpha_{i}^{2} \frac{\partial \tau_{i}}{\partial l_{\mathrm{r}, k_{1}}} \frac{\partial \tau_{i}}{\partial l_{\mathrm{r}, k_{2}}}\right)$

From (15), the contribution of the channel attenuation factors and time delays can be observed individually. Namely, the first and the second elements in (15) are related to the location information obtained from the channel attenuation factors and the time delay parameters, respectively. Hence, it is noted that both RSS and TOA parameters are utilized for localization in the synchronous scenario.

\section{B. Direct Positioning}

Direct positioning refers to the estimation of the unknown location directly from the received signals without any intermediate steps for estimating location related parameters such as TOA or RSS [14], [15], [17]-[21], [29]. Direct positioning has not been considered before for synchronous VLP systems, which carry significant differences from RF based positioning systems.

In direct positioning, the aim is to estimate the location of the VLC receiver, $l_{\mathrm{r}}$, based on the received signals in (1). From (4) and (7), the ML estimator for $l_{\mathrm{r}}$ can be obtained as follows [28]:

$\hat{\boldsymbol{l}}_{\mathrm{r}}^{\mathrm{DP}, \mathrm{syn}}=\underset{\boldsymbol{l}_{\mathrm{r}}}{\arg \max }-\sum_{i=1}^{N_{\mathrm{L}}} \int_{T_{1, i}}^{T_{2, i}}\left(r_{i}(t)-\alpha_{i} R_{p} s_{i}\left(t-\tau_{i}\right)\right)^{2} d t$

which can be simplified, after some manipulation, into

$$
\begin{aligned}
\hat{\boldsymbol{l}}_{\mathrm{r}}^{\mathrm{DP}, \mathrm{syn}}=\underset{\boldsymbol{l}_{\mathrm{r}}}{\arg \max } & \sum_{i=1}^{N_{\mathrm{L}}} \alpha_{i} \int_{T_{1, i}}^{T_{2, i}} r_{i}(t) s_{i}\left(t-\tau_{i}\right) d t \\
& -\frac{R_{p}}{2} \sum_{i=1}^{N_{\mathrm{L}}} \alpha_{i}^{2} E_{2}^{i}
\end{aligned}
$$

where $E_{2}^{i}$ is as defined in (11). It should be noted that $\tau_{i}$ and $\alpha_{i}$ in (16) are functions of $l_{\mathrm{r}}$ as specified in (2) (with $\Delta_{i}=0$ ) and (3), respectively. Hence, the direct ML position estimator in (16) searches over all possible values of the unknown position $l_{\mathrm{r}}$ based on the relations of $l_{\mathrm{r}}$ with the channel attenuation factors and the time delays.

The main advantage of the direct positioning approach in (16) is related to its performance (optimality in the ML sense), as investigated in Section V. On the other hand, it can lead to high complexity in certain applications due to increased storage and communication requirements. For example, if the location estimation should be performance at a central unit, then it becomes cumbersome to transmit all the received signals to the center.

\section{Positioning in Asynchronous Systems}

In the asynchronous scenario, the VLC receiver is not synchronized with the LED transmitters; that is, $\Delta_{i}$ in (2) is a deterministic unknown parameter for each $i \in\left\{1, \ldots, N_{\mathrm{L}}\right\}$. In this section, the CRLB is derived for asynchronous VLP systems, and the direct position estimation and its relation to the two-step position estimation are investigated.

\section{A. $C R L B$}

In an asynchronous VLP system, the unknown parameters include the TOAs of the received signals coming from the LED transmitters in addition to the location of the VLC receiver. Hence, the vector of unknown parameters in (4) for the asynchronous case can be expressed as

$$
\boldsymbol{\varphi}=\left[\begin{array}{llllll}
l_{\mathrm{r}, 1} & l_{\mathrm{r}, 2} & l_{\mathrm{r}, 3} & \tau_{1} & \ldots & \tau_{N_{\mathrm{L}}}
\end{array}\right]^{T} .
$$

Then, the CRLB for estimating $\boldsymbol{l}_{\mathrm{r}}$ based on $r_{1}(t), \ldots, r_{N_{\mathrm{L}}}(t)$ in (1) is stated in the following proposition: 
Proposition 2: For asynchronous VLP systems, the CRLB on the MSE of any unbiased estimator $\hat{l}_{\mathrm{r}}$ for the location of the $V L C$ receiver is given by

$$
\mathbb{E}\left\{\left\|\hat{\boldsymbol{l}}_{\mathrm{r}}-\boldsymbol{l}_{\mathrm{r}}\right\|^{2}\right\} \geq \operatorname{trace}\left\{\mathbf{J}_{\text {asy }}^{-1}\right\}
$$

where $\mathbf{J}_{\text {asy }}^{-1}$ is a $3 \times 3$ matrix with the following elements:

$$
\left[\mathbf{J}_{\text {asy }}\right]_{k_{1}, k_{2}}=\frac{R_{p}^{2}}{\sigma^{2}} \sum_{i=1}^{N_{\mathrm{L}}}\left(E_{2}^{i}-\frac{\left(E_{3}^{i}\right)^{2}}{E_{1}^{i}}\right) \frac{\partial \alpha_{i}}{\partial l_{\mathrm{r}, k_{1}}} \frac{\partial \alpha_{i}}{\partial l_{\mathrm{r}, k_{2}}}
$$

for $k_{1}, k_{2} \in\{1,2,3\}$, with $E_{1}^{i}, E_{2}^{i}, E_{3}^{i}$, and $\partial \alpha_{i} / \partial l_{\mathrm{r}, k}$ being as defined in (10), (11), (12), and (14), respectively.

It is noted from the CRLB expression in Proposition 2 that the position related information in the channel attenuation factors (RSS) is utilized in the asynchronous case for estimating the location of the VLC receiver (see (19)). On the other hand, information from both the channel attenuation factors (RSS) and the time delay (TOA) parameters is available in the synchronous case as can be noted from Proposition 1. In addition, the CRLB expression presented in Proposition 2 has been obtained for the first time in the literature, which constitutes a novel contribution to localization in asynchronous VLP systems. Since the expression in (19) is derived based on the entire observation signal $r(t)$ in (1), it differs from the CRLB expression in [9], which derives the CRLB for RSS based localization based on the measured RSS values without directly using the received signal (equation (32), p.6). On the other hand, when $E_{3}^{i}=0$ for $i=1, \ldots, N_{\mathrm{L}}$, which is valid for many practical pulses, the FIM expression in (19) is equivalent to that in [9]. Hence, the CRLB provided by Proposition 2 also covers the more general case of $E_{3}^{i} \neq 0$ as compared to the CRLB in [9], which constitutes a special case of (19). Indeed, it is proved in Proposition 3 in Section IV-B that the direct positioning approach adopted for derivation of (19) is equivalent to the two-step method for asynchronous VLP systems under the condition $E_{3}^{i}=0$. This results explains the equivalence of the two expressions in (19) and [9] for practical localization scenarios.

Remark 1: From Proposition 1 and Proposition 2, it is observed that if $E_{3}^{i}=0$ and $\alpha_{i}^{2} E_{1}^{i} \ll E_{2}^{i}$ for $i=1, \ldots, N_{\mathrm{L}}$, the CRLB expressions in the synchronous and asynchronous cases converge to each other. This corresponds to scenarios in which the position related information in the time delay (TOA) parameters is negligible compared to that in the channel attenuation factors (RSS parameters). Hence, synchronism does not provide any significant benefits in such scenarios. Since $E_{1}^{i} / E_{2}^{i}$ can be expressed from Parseval's relation as $4 \pi^{2} \beta_{i}^{2}$, where $\beta_{i}$ is the effective bandwidth of $s_{i}(t),{ }^{2}$ it can be concluded that the synchronous and asynchronous cases lead to similar CRLBs when the transmitted signals have small effective bandwidths. This is an intuitive result because TOA information gets less accurate as the effective bandwidth decreases [5].

\footnotetext{
${ }^{2}$ The effective bandwidth is defined as $\beta_{i}=\sqrt{\left(1 / E_{2}^{i}\right) \int f^{2}\left|S_{i}(f)\right|^{2} d f}$, where $S_{i}(f)$ is the Fourier transform of $s_{i}(t)$.
}

\section{B. Direct and Two-Step Positioning}

Direct position estimation involves the estimation of $\boldsymbol{l}_{\mathrm{r}}$, the location of the VLC receiver, directly from the received signals in (1). From (4), the ML estimator for direct positioning in the asynchronous case can be obtained as follows:

$\hat{\varphi}_{\mathrm{ML}}=\underset{\varphi}{\arg \max } \sum_{i=1}^{N_{\mathrm{L}}}\left(\alpha_{i} \int_{T_{1, i}}^{T_{2, i}} r_{i}(t) s_{i}\left(t-\tau_{i}\right) d t-\frac{R_{p}}{2} \alpha_{i}^{2} E_{2}^{i}\right)$

where $\varphi$ is defined by (17), $\alpha_{i}$ is related to $\boldsymbol{l}_{\mathrm{r}}$ as in (3), and $E_{2}^{i}$ is given by (11). Since $\alpha_{i}$ 's are nonnegative and the integral expressions depend only on $\tau_{i}$ 's (20), the ML estimates for $\tau_{i}$ 's can be calculated as

$$
\hat{\tau}_{i}=\underset{\tau_{i}}{\arg \max } \int_{T_{1, i}}^{T_{2, i}} r_{i}(t) s_{i}\left(t-\tau_{i}\right) d t .
$$

Then, the ML estimate for $\boldsymbol{l}_{\mathrm{r}}$ is obtained from (20) as

$$
\hat{\boldsymbol{l}}_{\mathrm{r}}^{\mathrm{DP}, \text { asy }}=\underset{\boldsymbol{l}_{\mathrm{r}}}{\arg \max } \sum_{i=1}^{N_{\mathrm{L}}}\left(\alpha_{i} \tilde{C}_{r s}^{i}-0.5 R_{p} \alpha_{i}^{2} E_{2}^{i}\right)
$$

where $\tilde{C}_{r s}^{i}$ is defined as

$$
\tilde{C}_{r s}^{i} \triangleq \int_{T_{1, i}}^{T_{2, i}} r_{i}(t) s_{i}\left(t-\hat{\tau}_{i}\right) d t
$$

The following proposition states that the direct position estimator in (22) is equivalent to a two-step position estimator if $E_{3}^{i}=0$ for $i=1, \ldots, N_{\mathrm{L}}$.

Proposition 3: Consider an asynchronous VLP system with $E_{3}^{i}=0$ for $i=1, \ldots, N_{\mathrm{L}}$. Then, the direct position estimator in (22) is equivalent to the following two-step position estimator:

First step: Estimate $\tau_{i}$ based on $r_{i}(t)$ as in (21) and estimate $\alpha_{i} \overline{\text { as } \hat{\alpha}_{i}=} \tilde{C}_{r s}^{i} /\left(R_{p} E_{2}^{i}\right)$ for $i=1, \ldots, N_{\mathrm{L}}$, where $\tilde{C}_{r s}^{i}$ is as in (23).

Second step: Based on $\left\{\hat{\alpha}_{i}\right\}_{i=1}^{N_{\mathrm{L}}}$, estimate $\boldsymbol{l}_{\mathrm{r}}$ as

$$
\hat{\boldsymbol{l}}_{\mathrm{r}}^{\mathrm{TS}, \text { asy }}=\underset{\boldsymbol{l}_{\mathrm{r}}}{\arg \min } \sum_{i=1}^{N_{\mathrm{L}}} E_{2}^{i}\left(\hat{\alpha}_{i}-\alpha_{i}\right)^{2}
$$

where $\alpha_{i}$ is as defined in (3).

Proposition 3 implies that two-step position estimation is optimal in the ML sense for asynchronous VLP systems; that is, the direct positioning (based on ML estimation) is equivalent to the two-step positioning when $E_{3}^{i}=0$ for $i=1, \ldots, N_{\mathrm{L}}$. Since $s_{i}(0)=s_{i}\left(T_{s, i}\right)$ for many practical pulses, $E_{3}^{i}=0$ is encountered in practice (see (12)); hence, the two-step estimator can be employed in real systems as the optimal approach in the ML sense.

\section{Numerical Results}

In this section, numerical results are presented to validate the theoretical derivations in the previous sections. As in [11], the responsivity of the photo detector is taken as $R_{p}=0.4 \mathrm{~mA} / \mathrm{mW}$, and the spectral density level of the noise is set to $\sigma^{2}=1.336 \times 10^{-22} \mathrm{~W} / \mathrm{Hz}$. In addition, the Lambertian order is taken as $m=1$ and the area $S$ of the photo detector 
at the VLC receiver is equal to $1 \mathrm{~cm}^{2}$. The transmitted signal $s(t)$ in (1) is modeled as [11]

$$
s(t)=A\left(1-\cos \left(2 \pi t / T_{s}\right)\right)\left(1+\cos \left(2 \pi f_{c} t\right)\right) \mathbb{I}_{t \in\left[0, T_{s}\right]}
$$

where $f_{c}$ denotes the center frequency, $A$ corresponds to the average emitted optical power; that is, source optical power, and $\mathbb{I}_{t \in\left[0, T_{s}\right]}$ represents an indicator function, which is equal to 1 if $t \in\left[0, T_{s}\right]$ and zero otherwise. For the network configuration, consider a room with a width, depth and height of $\left[\begin{array}{lll}8 & 8 & 5\end{array}\right] \mathrm{m}$., respectively, where four LED transmitters are attached to the ceiling with positions $\boldsymbol{l}_{\mathrm{t}}^{1}=\left[\begin{array}{lll}2 & 2 & 5\end{array}\right]^{T} \mathrm{~m}$., $\boldsymbol{l}_{\mathrm{t}}^{2}=\left[\begin{array}{lll}6 & 2 & 5\end{array}\right]^{T} \mathrm{~m} ., \boldsymbol{l}_{\mathrm{t}}^{3}=\left[\begin{array}{lll}2 & 6 & 5\end{array}\right]^{T} \mathrm{~m}$. and $\boldsymbol{l}_{\mathrm{t}}^{4}=\left[\begin{array}{lll}6 & 6 & 5\end{array}\right]^{T} \mathrm{~m}$. The VLC receiver is located at $\boldsymbol{l}_{\mathrm{r}}=\left[\begin{array}{lll}4 & 4 & 1\end{array}\right]^{T} \mathrm{~m}$. and looks upwards, i.e., the orientation vector is given by $\boldsymbol{n}_{\mathrm{r}}=\left[\begin{array}{lll}0 & 0 & 1\end{array}\right]^{T}$.

In the first simulation, the effect of the LED orientation angle on the performance of synchronous and asynchronous positioning is investigated. The orientation vectors of the LEDs are arranged in such a way that the projection of the LED orientation vectors onto the $x-y$ plane is always on the diagonal line connecting the room corners. More specifically, for an LED orientation angle $\theta$, which is the angle the orientation vector makes with the $z$-axis (positive angles denote that the LED is directed towards the center of the room), the LED orientation vectors are given by $\boldsymbol{n}_{\mathrm{t}}^{1}=$

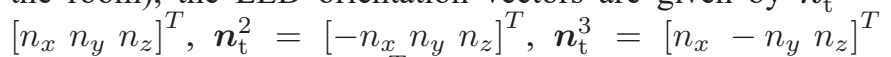
and $\boldsymbol{n}_{\mathrm{t}}^{4}=\left[-n_{x}-n_{y} n_{z}\right]^{T}$, where $n_{x}=\operatorname{sign}(\theta) \sin (\theta) / \sqrt{2}$, $n_{y}=\operatorname{sign}(\theta) \sin (\theta) / \sqrt{2}$ and $n_{z}=-\cos (\theta)$. Fig. 1 shows the CRLBs of synchronous and asynchronous positioning systems with respect to the LED orientation angle $\theta$, which are calculated based on Proposition 1 and Proposition 2, respectively. It is observed from Fig. 1 that the highest localization accuracy is achieved around $\theta=30^{\circ}$ for both systems, while the lineof-sight vector from the LED transmitter to the VLC receiver results in an angle of $35.3^{\circ}$ with the $z$-axis. From the CRLB expressions in (9) and (19), it can be inferred that the CRLBs depend on the SNR via the terms $R_{p}^{2} E_{2}^{i} / \sigma^{2}$ and $R_{p}^{2} \alpha_{i}^{2} E_{1}^{i} / \sigma^{2}$, and on the geometry via the terms $\partial \tau_{i} / \partial l_{\mathrm{r}, k}$ and $\partial \alpha_{i} / \partial l_{\mathrm{r}, k}$. Hence, although the highest SNR value is attained when the LEDs have the same orientation as the line-of-sight vector to the VLC receiver, the best localization performance is achieved at a different LED orientation due to the impact of the VLP system geometry. Furthermore, the positioning performance of the synchronous system exceeds that of the asynchronous one through the use of time delay information in the synchronous case.

In order to observe the localization performance throughout the entire room, the CRLBs for the synchronous VLP system are computed as the VLC receiver moves inside the room and the resulting contour plot is shown in Fig. 2. The configuration of the LEDs is the same as the first example except that the LED orientation angles are fixed at $\theta=0^{\circ}$. The CRLB values are obtained for position estimation of a VLC receiver with a fixed height $l_{\mathrm{r}, 3}=1 \mathrm{~m}$., moved along the $x-y$ plane over the room. As observed from Fig. 2, the localization performance decreases as the receiver moves away from the center of the room, which is an expected outcome since that movement leads to an increase in the distance, the incidence angle and

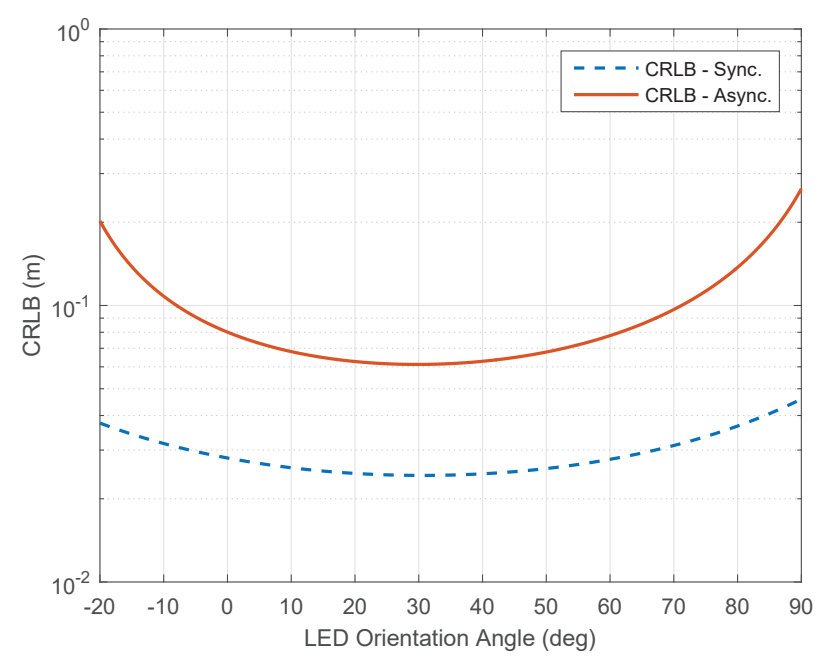

Fig. 1. CRLB versus LED orientation angle for synchronous and asynchronous VLP systems, where $T_{s}=0.1 \mathrm{~ms}, f_{c}=100 \mathrm{MHz}$, and $A=$ $100 \mathrm{~mW}$.

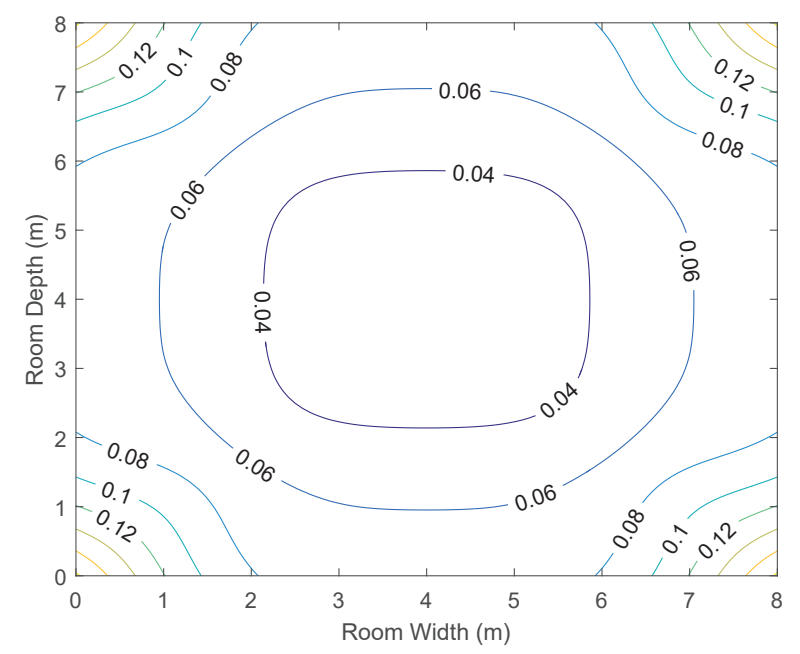

Fig. 2. CRLB for a synchronous VLP system as the VLC receiver moves inside the room, where $T_{s}=0.1 \mathrm{~ms}, f_{c}=100 \mathrm{MHz}$ and $A=100 \mathrm{~mW}$.

the irradiation angle between the VLC receiver and the LED transmitters, thereby reducing the signal strength, as implied by the Lambertian formula in (3).

Finally, in Fig. 3, the CRLBs and the MLEs for synchronous and asynchronous VLP systems are plotted with respect to the source optical power by setting $\theta=30^{\circ}$. It can be inferred from Fig. 3 that the accuracy of synchronous positioning outperforms that of asynchronous positioning since the former takes into account both the time delay information and the channel attenuation factor whereas the latter utilizes only the RSS information. Also, it is observed that the MLEs converge to the corresponding CRLBs at high SNR for both synchronous and asynchronous systems.

\section{Concluding Remarks}

In this paper, the CRLBs and MLEs have been derived for direct position estimation in synchronous and asynchronous 


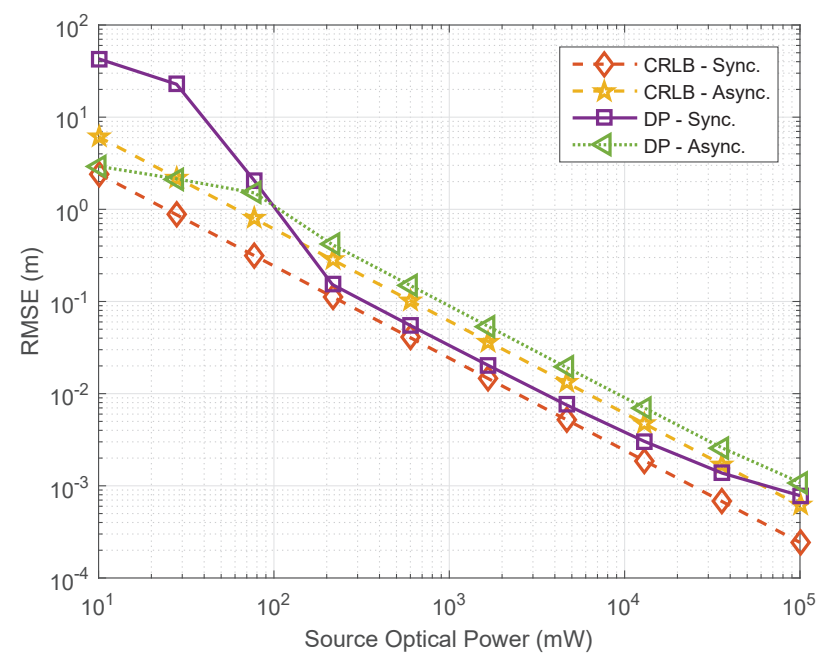

Fig. 3. CRLBs and MLEs for synchronous and asynchronous VLP systems with respect to the optical power, where $T_{s}=0.1 \mathrm{~ms}$ and $f_{c}=100 \mathrm{MHz}$.

VLP systems. The proposed CRLB expressions exploit the entire observation signal at the VLC receiver and can be applied to any VLP system where the LED transmitters and the VLC receiver have arbitrary orientations. Also, the CRLB on the localization accuracy of synchronous VLP systems, which utilizes both the TOA and RSS information, has been derived for the first time in the literature. In addition, the CRLB presented for the asynchronous case generalizes an expression available in the literature to any type of transmitted pulse. Comparative analysis on the performance of synchronous and asynchronous systems indicates that the advantage of synchronous positioning becomes more noticeable as the effective bandwidth of the transmitted pulse increases. Furthermore, it has been obtained that the direct and two-step estimators are equivalent for asynchronous VLP systems. Numerical results have been presented to illustrate the theoretical derivations.

\section{REFERENCES}

[1] P. H. Pathak, X. Feng, P. Hu, and P. Mohapatra, "Visible light communication, networking, and sensing: A survey, potential and challenges," IEEE Communications Surveys Tutorials, vol. 17, no. 4, pp. 2047-2077, Fourthquarter 2015.

[2] J. Armstrong, Y. Sekercioglu, and A. Neild, "Visible light positioning: A roadmap for international standardization," IEEE Communications Magazine, vol. 51, no. 12, pp. 68-73, Dec. 2013.

[3] Q.-L. Li, J.-Y. Wang, T. Huang, and Y. Wang, "Three-dimensional indoor visible light positioning system with a single transmitter and a single tilted receiver," Optical Engineering, vol. 55, no. 10, pp. $106103-1-$ $106103-7,2016$.

[4] W. Zhang, M. I. S. Chowdhury, and M. Kavehrad, "Asynchronous indoor positioning system based on visible light communications," Optical Engineering, vol. 53, no. 4, pp. 045 105-1-045 105-9, 2014

[5] Z. Sahinoglu, S. Gezici, and I. Guvenc, Ultra-Wideband Positioning Systems: Theoretical Limits, Ranging Algorithms, and Protocols. New York, Cambridge University Press, 2008

[6] M. Aminikashani, W. Gu, and M. Kavehrad, "Indoor positioning with OFDM visible light communications," in 13th IEEE Annual Consumer Communications Networking Conference (CCNC), Jan. 2016, pp. 505510 .

[7] Y. Eroglu, I. Guvenc, N. Pala, and M. Yuksel, "AOA-based localization and tracking in multi-element VLC systems," in IEEE 16th Annual Wireless and Microwave Technology Conference (WAMICON),, Apr. 2015.
[8] S.-H. Yang, H.-S. Kim, Y.-H. Son, and S.-K. Han, “Three-dimensional visible light indoor localization using AOA and RSS with multiple optical receivers," Journal of Lightwave Technology, vol. 32, no. 14 pp. 2480-2485, July 2014.

[9] A. Sahin, Y. S. Eroglu, I. Guvenc, N. Pala, and M. Yuksel, "Hybrid 3-d localization for visible light communication systems," Journal of Lightwave Technology, vol. 33, no. 22, pp. 4589-4599, Nov 2015.

[10] G. B. Prince and T. D. C. Little, "Latency constrained device positioning using a visible light communication two-phase received signal strength angle of arrival algorithm," in 2015 International Conference on Indoor Positioning and Indoor Navigation (IPIN), Oct 2015, pp. 1-7.

[11] T. Wang, Y. Sekercioglu, A. Neild, and J. Armstrong, "Position accuracy of time-of-arrival based ranging using visible light with application in indoor localization systems," Journal of Lightwave Technology, vol. 31 , no. 20, pp. 3302-3308, Oct. 2013.

[12] M. F. Keskin and S. Gezici, "Comparative theoretical analysis of distance estimation in visible light positioning systems," Journal of Lightwave Technology, vol. 34, no. 3, pp. 854-865, Feb 2016.

[13] S.-Y. Jung, S. Hann, and C.-S. Park, "TDOA-based optical wireless indoor localization using LED ceiling lamps," IEEE Transactions on Consumer Electronics, vol. 57, no. 4, pp. 1592-1597, Nov. 2011.

[14] A. J. Weiss and A. Amar, "Direct position determination of multiple radio signals," EURASIP Journal on Advances in Signal Processing, vol. 2005, no. 1, p. 653549, 2005.

[15] O. Bialer, D. Raphaeli, and A. J. Weiss, "Maximum-likelihood direct position estimation in dense multipath," IEEE Transactions on Vehicular Technology, vol. 62, no. 5, pp. 2069-2079, Jun 2013.

[16] A. Amar and A. J. Weiss, "New asymptotic results on two fundamental approaches to mobile terminal location," in 2008 3rd International Symposium on Communications, Control and Signal Processing, March 2008, pp. 1320-1323.

[17] P. Closasy, C. Fernndez-Pradesz, and J. A. Fernndez-Rubioy, "Direct position estimation approach outperforms conventional two-steps positioning," in 2009 17th European Signal Processing Conference, Aug 2009, pp. 1958-1962.

[18] N. Garcia, A. M. Haimovich, M. Coulon, and J. A. Dabin, "High precision TOA-based direct localization of multiple sources in multipath," CoRR, vol. abs/1505.03193, 2015

[19] N. Vankayalapati, S. Kay, and Q. Ding, "TDOA based direct positioning maximum likelihood estimator and the Cramer-Rao bound," IEEE Transactions on Aerospace and Electronic Systems, vol. 50, no. 3, pp. 1616-1635, July 2014

[20] O. Bar-Shalom and A. J. Weiss, "Direct positioning of stationary targets using MIMO radar,” Signal Processing, vol. 91, no. 10, pp. 2345-2358, 2011.

[21] L. Tzafri and A. J. Weiss, "High-resolution direct position determination using MVDR," IEEE Transactions on Wireless Communications, vol. 15, no. 9, pp. 6449-6461, Sept 2016.

[22] H. Steendam, T. Q. Wang, and J. Armstrong, "Cramer-Rao bound for indoor visible light positioning using an aperture-based angular-diversity receiver," in 2016 IEEE International Conference on Communications (ICC), May 2016, pp. 1-6.

[23] G. Kail, P. Maechler, N. Preyss, and A. Burg, "Robust asynchronous indoor localization using led lighting," in 2014 IEEE International Conference on Acoustics, Speech and Signal Processing (ICASSP), May 2014, pp. 1866-1870.

[24] X. Zhang, J. Duan, Y. Fu, and A. Shi, "Theoretical accuracy analysis of indoor visible light communication positioning system based on received signal strength indicator," Journal of Lightwave Technology, vol. 32, no. 21, pp. 4180-4186, Nov. 2014.

[25] D. Karunatilaka, F. Zafar, V. Kalavally, and R. Parthiban, "LED based indoor visible light communications: State of the art," IEEE Communications Surveys \& Tutorials, 2015.

[26] Y. Qi and H. Kobayashi, "Cramér-Rao lower bound for geolocation in non-line-of-sight environment," in IEEE International Conference on Acoustics, Speech, and Signal Processing (ICASSP), vol. 3, May 2002, pp. III-2473-III-2476.

[27] N. Decarli and D. Dardari, "Ziv-Zakai bound for time delay estimation of unknown deterministic signals," in IEEE International Conference on Acoustics Speech and Signal Processing (ICASSP), May 2014, pp. 4673-4677.

[28] H. V. Poor, An Introduction to Signal Detection and Estimation. New York: Springer-Verlag, 1994.

[29] A. J. Weiss, "Direct position determination of narrowband radio frequency transmitters," IEEE Signal Processing Letters, vol. 11, no. 5, pp. 513-516, May 2004. 